\title{
Prospective association between consumption frequency of organic food and body weight change, risk of overweight or obesity: results from the NutriNet-Santé Study
}

\author{
Emmanuelle Kesse-Guyot ${ }^{1 *}$, Julia Baudry ${ }^{1}$, Karen E. Assmann ${ }^{1}$, Pilar Galan ${ }^{1}$, Serge Hercberg $^{1,2}$ \\ and Denis Lairon ${ }^{3}$ \\ ${ }^{1}$ Sorbonne Paris Cité Epidemiology and Statistics Research Center (CRESS), National Institute for Health and Medical Research \\ (INSERM, U1153), National Institute for Agricultural Research (INRA, U1125), National Conservatory of Arts and Crafts \\ (CNAM), Nutritional Epidemiology Research Team (EREN), Paris 13 University, F-93017 Bobigny, France \\ ${ }^{2}$ Département de Santé Publique, Hôpital Avicenne, F-93017 Bobigny, France \\ ${ }^{3}$ Nutrition, Obésité et Risque Thrombotique (NORT), National Institute for Health and Medical Research (INSERM), UMR S \\ 1062, National Institute for Agricultural Research (INRA) 1260, Aix Marseille University, F-13385 Marseille, France
}

(Submitted 12 September 2016 - Final revision received 19 December 2016 - Accepted 5 January 2017 - First published online 7 Febraury 2017)

\section{Abstract}

A lower BMI has been reported among consumers of organic foods, but this relationship has never been examined in a prospective design study. Our aim was to prospectively investigate the association between frequency of organic food consumption and weight change. We analysed data from 62224 participants of the NutriNet-Santé cohort ( $78 \%$ women, mean age $=45$ years) with information on consumption frequency of organic foods, dietary intake and repeated anthropometric data. For sixteen products, participants reported their consumption frequency of labelled organic foods (never, occasionally, most of the time). An organic score (OS) with a maximum of thirty-two points was computed. The associations of the OS (modeled as quartiles (Q)) with change in BMI during follow-up (on average $3 \cdot 1$ years) and with the risk of overweight and obesity were estimated by ANCOVA and multivariable logistic regression. A lower BMI increase was observed across quartiles of the OS (mean difference Q4 $v$. Q1 $=-0 \cdot 16(95 \% \mathrm{CI}-0 \cdot 32,-0 \cdot 01)$. An increase in the OS was associated with a lower risk of overweight and obesity (among non-overweight and non-obese participants at inclusion): OR for Q4 $v$. Q1 were 0.77 (95\% CI 0.68, 0.86) and 0.69 (95\% CI $0.58,0.82)$, respectively. Concerning obesity risk, the association was stronger among participants with higher adherence to nutritional guidelines. This study supports a strong protective role of consumption frequency of organic foods with regard to the risk of overweight and obesity that depends on overall dietary quality. Upon confirmation, these results may contribute to fine-tune nutritional guidelines by accounting for farming practices in food production.

Key words: Organic foods: Obesity: Prospective cohort studies: Weight gain

The global obesity epidemic, involving multifaceted origins, is a major public health issue ${ }^{(1)}$. Overweight and obesity contribute to the global burden of chronic diseases ${ }^{(2)}$ - especially type 2 diabetes and ischaemic $\mathrm{CVD}^{(3)}$ along with cancers ${ }^{(4,5)}$.

Among the factors involved in the aetiology of obesity, excess energy consumption, sedentary lifestyle and genetic susceptibility are well recognised, but there is growing concern about the identification of novel factors involved, such as gut microbiota $^{(6)}$ or environmental chemicals ${ }^{(7-10)}$.

Among the different dietary factors, organic food is of major interest, as it presents multiple features that could potentially protect from weight gain and obesity. Notably, compared with conventional food, organic food has been suggested to present better nutritional values concerning fatty acids profiles and specific micronutrients ${ }^{(11-13)}$. Nonetheless, the possible implications at an individual level in terms of daily nutrient intake are unknown because of the lack of food composition tables accounting for farming practices. A small number of clinical studies have been conducted that compared specific nutritional biomarkers according to the type of diet (organic or conventional). However, owing to short study durations, these studies were not well equipped to provide reliable results and findings were inconsistent ${ }^{(14)}$.

Organic foods are also characterised by markedly low levels or absence of pesticide residues, as repeatedly reported in food residue analyses ${ }^{(11,14,15)}$ and in experimental studies showing that adopting an organic diet leads to a drastic reduction in pesticide residues and urine metabolites in children and

Abbreviations: PNNS-GS, Programme National Nutrition Santé-guidelines score; mPNNS-GS, modified PNNS-GS.

* Corresponding author: E. Kesse-Guyot, email e.kesse@eren.smbh.univ-paris13.fr 
adults $^{(16-20)}$. Among most pesticide families (organochlorines (now banned in the EU but still persistent), organophosphates and pyrethrynoïds), a large number of molecules have been recognised as endocrine disruptors ${ }^{(21)}$, leading to possible metabolic disorders ${ }^{(22)}$. Indeed, a higher exposure to some of these compounds has been associated with a higher risk for obesity or type 2 diabetes in humans ${ }^{(9)}$.

In this context, consumption of organic foods might contribute to the management of weight gain and obesity risk. In a recent cross-sectional analysis based on the NutriNet-Santé cohort, we showed that participants identified as regular consumers of organic food, compared with non-consumers, presented reduced odds of being overweight or obese ( -36 and $-62 \%$ in men and -42 and $-48 \%$ in women, respectively) ${ }^{(23)}$. Comparable findings were reported in the German National Nutrition Survey II (NVS II), a nationwide food consumption study conducted among 13074 adults: German buyers of organic food exhibited healthier lifestyles compared with non-buyers and presented lower body weight ${ }^{(24)}$.

To the best of our knowledge, no epidemiological study has yet investigated the prospective relationship between the consumption frequency of organic foods and the risk of overweight and obesity. The main objective of the present study was thus to investigate the longitudinal association between the consumption frequency of organic foods and the change in BMI, the risk of overweight and the risk of obesity in a very large adult cohort. Given our previous finding that organic food consumers show a higher level of adherence to nutritional recommendations ${ }^{(23)}$ - especially higher consumption of fruits and vegetables and lower consumption of animal products a secondary objective was to explore a potential modifying effect of the nutritional quality of the diet on the investigated associations.

\section{Methods}

\section{Study population}

The NutriNet-Santé is a web-based, prospective, observational cohort study that was launched in France in May 2009. The objectives, design and methodology have been described elsewhere $^{(25)}$. The study was conducted according to the guidelines laid down in the Declaration of Helsinki, and was approved by the Institutional Review Board of the French Institute for Health and Medical Research (IRB Inserm no. 0000388FWA00005831) and the 'Commission Nationale de l'Informatique et des Libertés' (CNIL no. 908450 and no. 909216). All subjects signed an electronic informed consent.

\section{Data collection and computation}

Volunteers completed self-administrated questionnaires using a dedicated website at baseline and during follow-up on an approximately monthly basis. The baseline questionnaires enquired about socio-demographic data, lifestyle factors, health status, physical activity, anthropometrics and diet. These questionnaires were first pilot-tested and then compared with traditional assessment methods or objectively validated ${ }^{(26-28)}$.
Consumption frequency of organic products data. After enrolment of 2 months, volunteers were asked to provide information on their consumption frequency of sixteen labelled organic products (fruits, vegetables, soya, dairy products, meat and fish, eggs, grains and legumes, bread and cereals, flour, vegetable oils and condiments, ready-to-eat meals, coffee/tea/ herbal tea, wine, biscuits/chocolate/sugar/marmalade, other foods, dietary supplements). Initially, data collection was related to research questions focused on reasons for nonconsumption. Consumption frequencies were presented in eight modalities: (1) most of the time, (2) occasionally, (3) never (too expensive), (4) never (product not available), (5) never (I'm not interested in organic products), (6) never (I avoid such products), (7) never (for no specific reason) and (8) I don't know. For each product, we allocated two points and one point to the 'most of the time' and 'occasionally' modalities, respectively (and 0 otherwise), as the objective of the present study was to focus on the level of frequency and not on reasons for non-consumption. The sixteen dietary components were summed up to provide an organic score (ranging from 0 to 32).

Anthropometric data. At enrolment and yearly thereafter, participants were asked to report their weight and height assessed during a medical or occupational health examination by a physician or report self-measurements obtained using standardised procedures (on flat surface, lightly dressed and without shoes). Self-reported anthropometric data have been shown to present an elevated concordance with clinical assessment $^{(26)}$.

BMI $\left(\mathrm{kg} / \mathrm{m}^{2}\right)$ was calculated as weight divided by the square of height. Subjects were classified as underweight or normal weight (BMI $<25$ ), overweight (including obesity; BMI $\geq 25$ ) or obese (BMI $\geq 30$ ) according to the World Health Organization reference values ${ }^{(1)}$.

Dietary data and physical activity. At baseline, quantitative dietary intakes were assessed using three 24-h records (24HR), randomly allocated over a 2-week period, including 2 weekdays and 1 weekend day, using a validated method ${ }^{(27,28)}$. Participants reported all foods and beverages consumed at each eating occasion. Portion sizes were estimated with the help of photographs, derived from a previously validated picture booklet ${ }^{(29)}$ or directly entered as grams, volumes or purchased units. As alcohol was only episodically consumed by most individuals, alcohol intake was calculated using either the $24 \mathrm{HR}$ or a frequency questionnaire for those identified as abstainers from the $324 \mathrm{HR}$ days. Moreover, as fish and seafood are infrequently consumed by many individuals, weekly consumption of this food group was assessed by a specific frequency question. Individual daily mean food consumption was calculated from the $324 \mathrm{HR}$ and weighted for the type of day (weekday or weekend day). Nutrient intakes were calculated using the NutriNet-Santé composition table ${ }^{(30)}$. Under-reporters were identified and excluded using the validated method developed by Black $^{(31)}$. To assess nutritional diet quality, a modified version of the validated Programme National Nutrition Santé-guidelines score (PNNS-GS) (without physical 
activity) was computed. This modified score, the mPNNS-GS, reflects adherence to the official French nutritional recommendations ${ }^{(32)}$. The score includes twelve components: eight refer to food-serving recommendations (fruits and vegetables, starchy foods, wholegrain products, dairy products, meat, eggs and fish, fish and seafood, vegetable fat, water $v$. soda) and four refer to moderation in consumption (added fat, salt, sweets, alcohol). Moreover, points are deducted for overconsumption of salt, added sugars or when energy intake exceeds the estimated energy needs by more than $5 \%$.

In order to account for a posteriori dietary patterns as well, we performed a principal component analysis (PCA) on thirty-one aggregated food groups. Dietary patterns obtained by PCA are independent linear combinations of the thirty-one food group consumptions, maximising the explained variance. Two dietary patterns were retained on the basis of Cattel's Scree plots and the interpretability of the factors. The online Supplementary Table S1 presents all factor-loading coefficients (corresponding to the correlations between the different food groups and the two dietary patterns) $>0 \cdot 3$. For each participant, the individual pattern score was calculated by summing the intake of the thirty-one food groups, weighted by their factor loading.

Covariates. At baseline, self-administered questionnaires were used to collect data including age, sex, formal education ( $\leq$ high school diploma, high school, post-secondary graduate), occupation (managerial staff, intermediate profession, employee/ manual worker, retired, unemployed, never employed/homemaker and self-employed), marital status (cohabiting or single), income, number of children and smoking status (never, former and current). Income per household unit was calculated by dividing the household's total monthly income by the number of consumption units (CU), using the following coefficients: $1 \mathrm{CU}$ for the first adult in the household, $0.5 \mathrm{CU}$ for all other household members aged 14 years or older and $0.3 \mathrm{CU}$ for children under 14 years $^{(33)}$. The following categories of monthly income were used: <1200, 1200-1800, 1800-2700 and $>2700$ euros per household unit.

Physical activity was assessed by the International Physical Activity Questionnaire (IPAQ) ${ }^{(34)}$. Metabolic equivalents measured in $\mathrm{min} /$ week were computed. The recommended IPAQ categories of physical activity were used: low ( $<30 \mathrm{~min}$ of brisk walking/d), moderate (30-60 min of brisk walking/d or equivalent) and high ( $\geq 60$ min of brisk walking/d or equivalent).

The enquired baseline health data included use of medication and self-reported history of diseases (cancer, CVD, hypertension, dyslipidaemia and diabetes).

\section{Statistical analysis}

For the present study, we used data from volunteers who were included before June 2014 and initially aged 18-74 years. Among them, we selected those: (1) who completed the organic questionnaire, (2) with baseline anthropometric data and at least one measurement during follow-up, (3) with available data to compute PNNS-GS and (4) who were not identified as energy under-reporters (Fig. 1). Follow-up anthropometric data were collected until June 2015.

The participants included ( $N$ 62 224) in our analyses were compared with those excluded, using Mann-Whitney $U$ tests and $\chi^{2}$ tests.

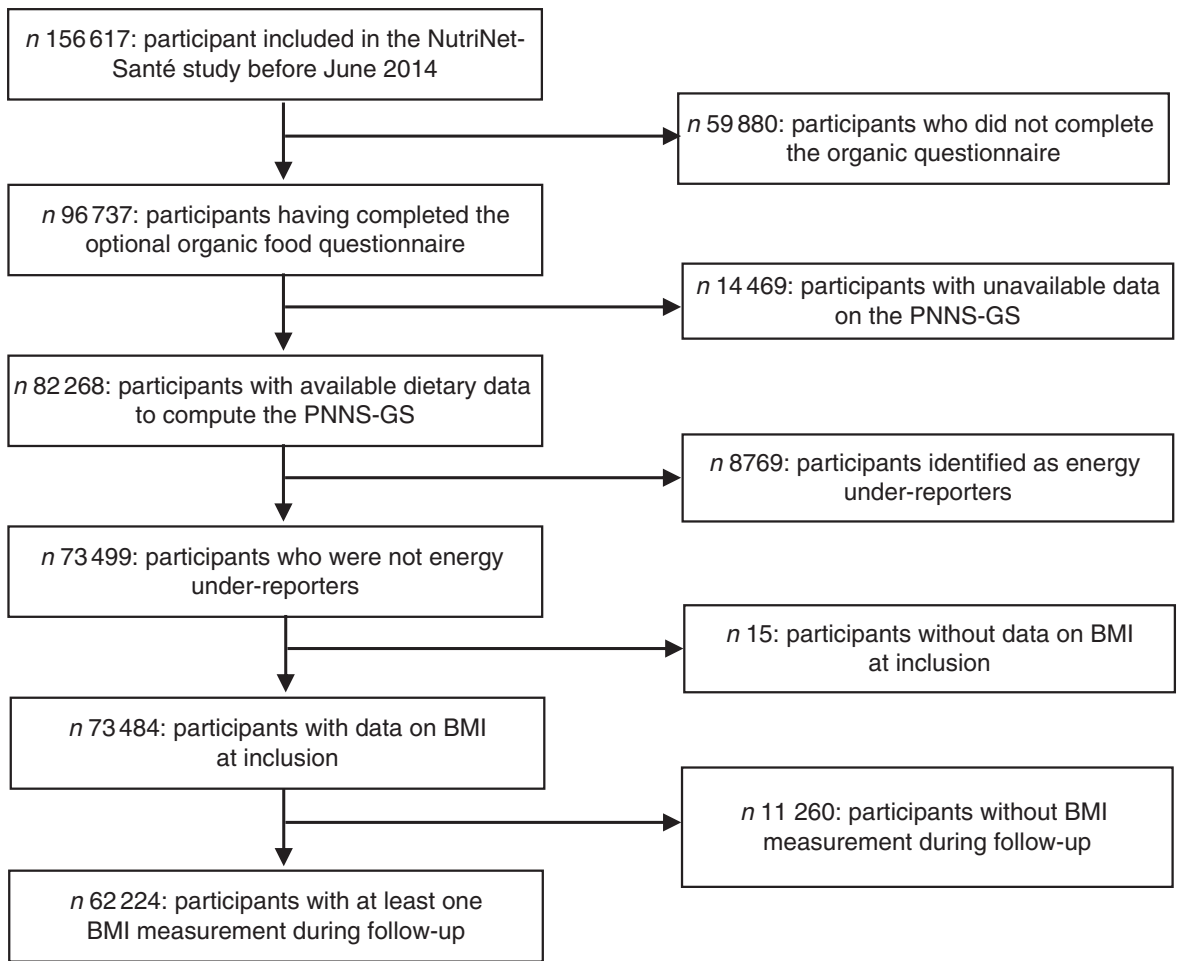

Fig. 1. Participants of the NutriNet-Santé selected for the present analyses, 2009-2015. PNNS-GS, Programme National Nutrition Santé-guidelines score. 
Baseline characteristics are presented by quartiles (Q) of the organic score. Values represent mean values and standard deviations or percentages, and $P$ values were calculated using linear contrast tests (for continuous variables) or $\chi^{2}$ trend tests (for categorical variables).

The association of the organic score with BMI change was assessed by ANCOVA, modelling change in BMI as a percentage of the baseline value. Mean differences and 95\% CI were presented across quartiles of the organic score. Three different models were run. The first model was adjusted for baseline age and sex. The second model was further adjusted for year and month of inclusion, follow-up duration, occupation, marital status, education, monthly income per household unit, baseline use of dietary supplements, the mPNNS-GS, PCA-extracted dietary patterns scores, energy intake, physical activity and smoking status. The final model was further adjusted for history of diseases (cancer, CVD, diabetes, hypertension and dyslipidaemia).

In a second set of analyses, we estimated OR and 95\% CI for becoming overweight or obese after exclusion of overweight and obese subjects at baseline, respectively (leading to new study samples of $n 43301$ and $n$ 56806). Three multivariate logistic regression models were computed with similar covariables to those used in the ANCOVA.

A set of supplementary analyses was performed for the obesity risk outcome. First, stratified analyses were conducted according to physical activity level/d (brisk walking $v$. $\geq 30 \mathrm{~min} / \mathrm{d}$ brisk walking or equivalent), use of dietary supplements (yes $v$. no), tobacco status (never and former smokers $v$. current smokers), education level and level of adherence to nutritional guidelines (using tertiles of the mPNNS-GS). Second, we used an alternative method of accounting for potential confounder bias: adjustment for a 'propensity score' that contains information on potential confounders in a combined manner ${ }^{(35)}$. To obtain the propensity score, a multinomial logistic regression model was used to estimate the predicted probability of organic food consumption (using quartiles of the sixteen-point organic score) as a function of a wide range of factors (socio-demographic, health characteristics, food group consumptions). Finally, we used inverse probability weighting to correct the estimates for potential selection bias ${ }^{(36)}$. All tests of statistical significance were two-sided, and the type I error was set at 5\%. Statistical analyses were performed using SAS $^{\circledR}$ software (version 9.3; SAS Institute Inc.).

\section{Results}

\section{Comparison of included and excluded participants}

Compared with excluded subjects, included subjects ( $N$ 62 224) were older, had a higher education level and income, were less often smokers and less physically active. They also presented a lower BMI and a higher nutritional quality of the diet (all $P$ values $<0 \cdot 05$, data not tabulated).

\section{Baseline Characteristics of the sample}

Baseline characteristics across quartiles of the organic score are shown in Table 1. Higher levels of the organic score were related to higher proportions of women, participants who were cohabiting, former smokers, physically active participants, individuals with post-secondary education and participants with high income or occupational level. A positive association was also observed with age, follow-up duration and the mPNNS-GS (reflecting the nutritional quality of the diet), whereas there was a negative association with energy intake, alcohol consumption and BMI.

Components of the organic score across quartiles of the organic score are shown in Table 2. In the first quartile (Q1), participants mostly reported no consumption of any organic products. In the fourth quartile (Q4), participants reported consuming organic products more frequently, especially products of the following food groups: eggs, starchy food, vegetables, vegetable oil, fruits and flour.

The mean follow-up time in our study sample was $3 \cdot 12$ $(\mathrm{SD}=1.37)$ years. Results on the prospective association between the organic score and the change in BMI over time are presented in Table 3. In the second model, higher organic scores were related to a substantially lower increase in BMI over time (mean difference Q4 $v$ Q $1=-0.15$ (95\% CI -0.31, -0.01), $\left.P_{\text {for trend }}=0 \cdot 05\right)$. After further adjustment for history of chronic diseases (third model), an even stronger association was observed (mean difference Q4 $v$. Q1 $=-0.16$ (95\% CI $-0 \cdot 32$, -0.01), $P_{\text {for trend }}=0.04$ ).

Results on the prospective association between the organic score and the risk of overweight and obesity are presented in Table 4. In the fully adjusted model, accounting for sociodemographic data, lifestyle and history of chronic diseases, a linear decrease in the risk of overweight was observed across quartiles of the organic score, with a risk reduction of $23 \%$ in Q4 compared with Q1. Findings concerning the risk of obesity were similar, with a risk reduction of $31 \%$ in Q4 compared with Q1.

Stratified analyses are presented in Fig. 2 and in the online Supplementary Table S2. We observed that the association between the organic score and the risk of obesity was stronger among participants with a higher nutritional quality of diet. Overall, in the different stratified analyses, the association between the organic score and the risk of obesity was observed in each subgroup, except for dietary supplement users and participants with an intermediate education level. Accounting for selection bias via inverse probability weighting did not substantially modify our findings (data not shown). Models with additional adjustment for a propensity score are presented in the online Supplementary Table S3. In this case, the investigated associations were attenuated but remained statistically significant.

\section{Discussion}

The results of the present study show, for the first time, a strong negative association between consumption frequency of organic foods and BMI change over time, as well as a marked reduction in the risk of overweight and obesity.

In the stratified analyses, significant associations were observed in almost all investigated subgroups, except for dietary supplement users and participants with an intermediate level of education. Importantly, both participants with a low level of physical activity and participants with a low level of education presented a significantly lower risk of obesity with increasing organic food 
Table 1. Baseline characteristics of the sample across organic score quartiles (Q), NutriNet-Santé study, 2009-2014, N 62224* (Mean values and standard deviations; percentages)

\begin{tabular}{|c|c|c|c|c|c|c|c|c|c|}
\hline & \multicolumn{2}{|c|}{ Q1 } & \multicolumn{2}{|c|}{ Q2 } & \multicolumn{2}{|c|}{ Q3 } & \multicolumn{2}{|c|}{ Q4 } & \multirow[b]{2}{*}{$P+$} \\
\hline & Mean & SD & Mean & SD & Mean & SD & Mean & SD & \\
\hline$n$ & \multicolumn{2}{|c|}{15245} & \multicolumn{2}{|c|}{16249} & \multicolumn{2}{|c|}{15807} & \multicolumn{2}{|c|}{14923} & \\
\hline Organic score $\max =32$ & 0.73 & 0.82 & 4.96 & 1.41 & $10 \cdot 36$ & 1.68 & $19 \cdot 15$ & $4 \cdot 21$ & $<0.0001$ \\
\hline Female (\%) & \multicolumn{2}{|c|}{73.45} & \multicolumn{2}{|c|}{77.94} & \multicolumn{2}{|c|}{$78 \cdot 71$} & \multicolumn{2}{|c|}{81.63} & $<0.0001$ \\
\hline Age (years) & $44 \cdot 16$ & $15 \cdot 34$ & $44 \cdot 36$ & 14.83 & $45 \cdot 62$ & $14 \cdot 29$ & $46 \cdot 72$ & $13 \cdot 29$ & $<0.0001$ \\
\hline Follow-up duration (d) & $1140 \cdot 34$ & 504.64 & $1157 \cdot 01$ & $500 \cdot 06$ & $1144 \cdot 88$ & $501 \cdot 80$ & $1118 \cdot 12$ & 499.69 & $<0.0001$ \\
\hline Education (\%) & & & & & & & & & $<0.0001$ \\
\hline Unidentified & \multicolumn{2}{|c|}{0.68} & \multicolumn{2}{|c|}{0.66} & \multicolumn{2}{|c|}{0.58} & \multicolumn{2}{|c|}{0.82} & \\
\hline$<$ High school diploma & \multicolumn{2}{|c|}{23.68} & \multicolumn{2}{|c|}{20.09} & \multicolumn{2}{|c|}{$17 \cdot 21$} & & & \\
\hline High school diploma & & & & & & & & & \\
\hline Post-secondary graduate & & & & & & & & & \\
\hline Monthly income per household unit in euros (\%) & & & & & & & & & $<0.0001$ \\
\hline Missing & 12 & & & & & & & 35 & \\
\hline $900-1200$ & 19 & & & & & & & & \\
\hline $1200-1800$ & 26 & & & & & & & & \\
\hline $1800-2700$ & 21 & & & & & & & & \\
\hline$>2700$ & 20 & & & & & & & & \\
\hline Occupational categories (\%) & & & & & & & & & $<0.0001$ \\
\hline Unemployed & & & & & & 18 & & 34 & \\
\hline Retired & 21 & & & & & & & & \\
\hline Employee/manual worker & & & & & & & & & \\
\hline Intermediate profession & & & & & & & & & \\
\hline Managerial staff & & & & & & & & & \\
\hline Never employed & 13 & & & & & & & & \\
\hline Craftsman, shopkeeper, business owner, farmer & & & & & & 68 & & 34 & \\
\hline Dietary supplement use (\%) & 35 & & & & & & & & $<0.0001$ \\
\hline Cohabiting (\%) & 80 & & & & & & & & $<0.0001$ \\
\hline Tobacco status (\%) & & & & & & & & & $<0.0001$ \\
\hline Former smoker & 33 & & & & & & & & \\
\hline Current smoker & 15 & & & & & & & & \\
\hline Never smoker & 51 & & & & & & & & \\
\hline Physical activity (\%) & & & & & & & & & $<0.0001$ \\
\hline Missing & 15 & & & & & & & & \\
\hline Low & & & & & & & & & \\
\hline Medium & & & & & & & & & \\
\hline High & 33 & & & & & & & & \\
\hline Energy intake (kJ/d) & $8125 \cdot 33$ & $2153 \cdot 71$ & $8011 \cdot 35$ & 2041.45 & $7985 \cdot 16$ & $2056 \cdot 10$ & 7925.50 & $1985 \cdot 30$ & $<0.0001$ \\
\hline Energy intake (kcal/d) & $1942 \cdot 90$ & $514 \cdot 75$ & $1914 \cdot 76$ & 487.92 & $1908 \cdot 50$ & 491.42 & $1894 \cdot 24$ & 474.50 & \\
\hline$\%$ Carbohydrates & 42.59 & 6.89 & 42.93 & $6 \cdot 81$ & 43.05 & 6.94 & 43.43 & $7 \cdot 11$ & $<0.0001$ \\
\hline$\%$ Lipids & 39.22 & $6 \cdot 61$ & 38.95 & $6 \cdot 46$ & 38.96 & 6.58 & 39.45 & $6 \cdot 72$ & 0.0017 \\
\hline$\%$ Proteins & $17 \cdot 87$ & 3.95 & $17 \cdot 80$ & 3.92 & $17 \cdot 67$ & $4 \cdot 01$ & $16 \cdot 81$ & 3.92 & $<0.0001$ \\
\hline Alcohol consumption $(\mathrm{g} / \mathrm{d})$ & 8.65 & $14 \cdot 11$ & $8 \cdot 27$ & $13 \cdot 16$ & $8 \cdot 22$ & $12 \cdot 23$ & 7.63 & $11 \cdot 20$ & $<0.0001$ \\
\hline mPNNS-GS & $7 \cdot 60$ & 1.62 & $7 \cdot 87$ & 1.62 & $8 \cdot 15$ & 1.60 & 8.44 & 1.58 & $<0.0001$ \\
\hline BMI $\left(\mathrm{kg} / \mathrm{m}^{2}\right)$ at baseline & $24 \cdot 50$ & 4.87 & 23.96 & 4.56 & $23 \cdot 71$ & $4 \cdot 31$ & 23.00 & 3.90 & $<0.0001$ \\
\hline Obesity (\%) at baselineł & & & & & & & & & $<0.0001$ \\
\hline BMI $\left(\mathrm{kg} / \mathrm{m}^{2}\right)$ at follow-up & $24 \cdot 72$ & 4.92 & $24 \cdot 17$ & 4.62 & 23.91 & $4 \cdot 40$ & $23 \cdot 13$ & 4.00 & $<0.0001$ \\
\hline Obesity (\%) at follow-upł & 12 & & & & & & & & $<0.0001$ \\
\hline
\end{tabular}

mPNNS-GS, modified Programme National Nutrition Santé-guidelines score.

* All variables were assessed at baseline, except when listed as 'at follow-up'.

$\dagger P$ for linear contrast.

$\ddagger \mathrm{BMI} \geq 30 \mathrm{~kg} / \mathrm{m}^{2}$.

consumption. It is noteworthy that the nutritional quality of the diet, estimated using an a priori dietary index reflecting adherence to the French nutritional guidelines ${ }^{(32)}$, appears to be a key effect modifier. Indeed, the strongest associations were observed among participants with high-nutritional-quality diets.

\section{Consumption frequency of organic foods and the risk of obesity or overweight}

We prospectively observed a markedly lower risk of obesity among subjects with a high consumption frequency of organic foods. Previous investigations of data from the NutriNet-Santé cohort have revealed that regular consumers of organic foods had healthier diets and healthier lifestyle characteristics (related to physical activity and tobacco use) as compared with irregular consumers or non-consumers. Moreover, regular consumers of organic foods in the NutriNet-Santé study presented specificities with respect to chronic disease history ${ }^{(23,37)}$. After accounting for these potential confounders, the findings of the present study were partially attenuated, but the association remained strong and highly significant, with a reduction in the risk of obesity of $37 \%$ after a 3.1-year follow-up.

A similar association was observed for overweight, although the strength of the association was smaller. 
Table 2. Components of the organic score across organic score quartiles (Q), NutriNet-Santé study, $N 62224^{*}$ (Mean values and standard deviations)

\begin{tabular}{|c|c|c|c|c|c|c|c|c|c|}
\hline & \multicolumn{2}{|c|}{ Q1 } & \multicolumn{2}{|c|}{ Q2 } & \multicolumn{2}{|c|}{ Q3 } & \multicolumn{2}{|c|}{ Q4 } & \multirow[b]{2}{*}{$P \dagger$} \\
\hline & Mean & SD & Mean & SD & Mean & SD & Mean & SD & \\
\hline Fruits & 0.06 & 0.25 & 0.57 & 0.52 & 0.95 & 0.41 & 1.49 & 0.53 & $<0.0001$ \\
\hline Vegetables & 0.07 & 0.27 & 0.59 & 0.55 & 0.97 & 0.48 & 1.51 & 0.54 & $<0.0001$ \\
\hline Rice, pasta, other cereals & 0.06 & 0.23 & 0.42 & 0.51 & 0.90 & 0.50 & 1.62 & 0.52 & $<0.0001$ \\
\hline Breads & 0.05 & 0.22 & 0.36 & 0.51 & 0.82 & 0.53 & 1.44 & 0.59 & $<0.0001$ \\
\hline Flour & 0.04 & 0.20 & 0.23 & 0.45 & 0.67 & 0.62 & 1.47 & 0.65 & $<0.0001$ \\
\hline Vegetable oils & 0.04 & 0.22 & 0.27 & 0.51 & 0.74 & 0.65 & 1.58 & 0.58 & $<0.0001$ \\
\hline Dairy products & 0.05 & 0.23 & 0.40 & 0.54 & 0.86 & 0.58 & 1.42 & 0.65 & $<0.0001$ \\
\hline Meats and seafoods & 0.02 & 0.14 & 0.19 & 0.41 & 0.50 & 0.54 & 0.92 & 0.66 & $<0.0001$ \\
\hline Eggs & 0.13 & 0.36 & 0.70 & 0.70 & $1 \cdot 19$ & 0.70 & 1.72 & 0.54 & $<0.0001$ \\
\hline Soya product & 0.06 & 0.24 & 0.20 & 0.44 & 0.40 & 0.59 & 0.88 & 0.76 & $<0.0001$ \\
\hline Sweet product & 0.03 & 0.19 & 0.33 & 0.48 & 0.76 & 0.50 & 1.38 & 0.58 & $<0.0001$ \\
\hline Tea, coffee, herbal tea & 0.04 & $0 \cdot 19$ & 0.27 & 0.47 & 0.66 & 0.59 & 1.33 & 0.67 & $<0.0001$ \\
\hline Wines & 0.03 & $0 \cdot 17$ & $0 \cdot 14$ & 0.35 & 0.29 & 0.47 & 0.65 & 0.64 & $<0.0001$ \\
\hline Ready-to-use dishes & 0.01 & 0.08 & 0.06 & 0.24 & 0.19 & 0.39 & 0.39 & 0.54 & $<0.0001$ \\
\hline Dietary supplements & 0.03 & $0 \cdot 18$ & 0.13 & 0.36 & 0.25 & 0.49 & 0.56 & 0.68 & $<0.0001$ \\
\hline Other dietary items & 0.01 & 0.12 & 0.09 & 0.30 & 0.24 & 0.45 & 0.80 & 0.80 & $<0.0001$ \\
\hline
\end{tabular}

* Subscores, with non-consumption, occasional consumption and regular consumption are coded 0,1 and 2 , respectively.

$\dagger P$ for linear trend.

Table 3. Association between organic scores in quartiles (Q) and BMI change over time, NutriNet-Santé study, 2009-2015, N 62224* (Mean differences and 95\% confidence intervals)

\begin{tabular}{|c|c|c|c|c|c|c|c|c|c|}
\hline \multirow[b]{2}{*}{ Models } & \multicolumn{2}{|c|}{ Q1 } & \multicolumn{2}{|c|}{ Q2 } & \multicolumn{2}{|c|}{ Q3 } & \multicolumn{2}{|c|}{ Q4 } & \multirow[b]{2}{*}{$P_{\text {for trend }}$} \\
\hline & Mean & $95 \% \mathrm{Cl}$ & Mean & $95 \% \mathrm{Cl}$ & Mean & $95 \% \mathrm{Cl}$ & Mean & $95 \% \mathrm{Cl}$ & \\
\hline Model 1† & 0.00 & Ref. & 0.01 & $-0.13,0.15$ & -0.06 & $-0.20,0.08$ & -0.34 & $-0.49,-0.20$ & $<0.0001$ \\
\hline Model $2 \ddagger$ & 0.00 & Ref. & 0.03 & $-0 \cdot 11,0 \cdot 17$ & 0.01 & $-0.13,0.16$ & -0.15 & $-0.31,-0.00$ & 0.05 \\
\hline Model $3 \S$ & 0.00 & Ref. & 0.03 & $-0.12,0.17$ & 0.01 & $-0.14,0.15$ & -0.16 & $-0.32,-0.01$ & 0.04 \\
\hline
\end{tabular}

Ref., referent values; mPNNS-GS, modified Programme National Nutrition Santé-guidelines score.

${ }^{*} A$ negative value $-0 \cdot(x, x)$ indicates that the observed increase (expressed as a percentage of the initial anthropometric marker) was lower than $0 \cdot(x, x)$ in the respective quartile than in Q1 (Ref.).

$\dagger$ Model 1 is adjusted for age and sex.

¥ Model 2 is model 1 further adjusted for month and year of inclusion, duration of follow-up, occupation, marital status, education, monthly income per unit, dietary supplement use, mPNNS-GS, principal component analysis-extracted dietary pattern scores, energy intake, physical activity and tobacco status.

$\S$ Model 3 is model 2 further adjusted for the history of chronic diseases.

Table 4. Prospective association between quartiles (Q) of the organic score and the risk of overweight or obesity, NutriNet-Santé study, 2009-2015* (Odds ratios and $95 \%$ confidence intervals using Q1 as the reference)

\begin{tabular}{|c|c|c|c|c|c|c|c|c|c|}
\hline & \multirow[b]{2}{*}{ Models } & \multirow[b]{2}{*}{ Q1 } & \multicolumn{2}{|c|}{ Q2 } & \multicolumn{2}{|c|}{ Q3 } & \multicolumn{2}{|c|}{ Q4 } & \multirow[b]{2}{*}{$P_{\text {for trend }}$} \\
\hline & & & OR & $95 \% \mathrm{Cl}$ & OR & $95 \% \mathrm{Cl}$ & OR & $95 \% \mathrm{Cl}$ & \\
\hline \multirow[t]{4}{*}{ Overweight } & (n 3259/40 042) & & & & & & & & \\
\hline & Model $1 \dagger$ & 1.00 & 0.94 & $0.86,1.04$ & 0.83 & $0.75,0.91$ & 0.62 & $0.56,0.69$ & $<\cdot 0001$ \\
\hline & Model 2‡ & 1.00 & 1.00 & $0.91,1.10$ & 0.93 & $0.84,1.02$ & 0.75 & $0.67,0.84$ & $<\cdot 0001$ \\
\hline & $\begin{array}{c}\text { Model 3§ } \\
(n 1337 / 55469)\end{array}$ & 1.00 & 1.00 & $0.91,1.10$ & 0.93 & $0.84,1.03$ & 0.77 & $0.68,0.86$ & $<.0001$ \\
\hline \multirow{3}{*}{ Obesity } & Model $1 \dagger$ & 1.00 & 0.87 & $0.75,1.01$ & 0.79 & $0.68,0.91$ & 0.52 & $0.45,0.61$ & $<.0001$ \\
\hline & Model $2 \ddagger$ & 1.00 & 0.93 & $0.80,1.08$ & 0.90 & $0.78,1.04$ & 0.66 & $0.55,0.78$ & $<.0001$ \\
\hline & Model $3 \S$ & 1.00 & 0.94 & $0.81,1.09$ & 0.92 & $0.79,1.06$ & 0.69 & $0.58,0.82$ & 0.0001 \\
\hline
\end{tabular}

mPNNS-GS, modified Programme National Nutrition Santé-guidelines score.

* Overweight (including obesity) and obesity analyses were performed among participants who were not overweight or obese at inclusion, respectively.

† Model 1 is adjusted for age and sex.

¥ Model 2 is model 1 further adjusted for month and year of inclusion, delay in follow-up, occupation, marital status, education, monthly income per unit, dietary supplement use, mPNNS-GS, principal component analysis-extracted dietary pattern scores, energy intakes, physical activity and tobacco status.

$\S$ Model 3 is model 2 further adjusted for the history of chronic diseases.

\section{Comparison of our results with the findings of other studies}

No previous longitudinal study has investigated the association between organic food consumption frequency and weight change or risk of overweight and obesity, but a potential beneficial link between body weight and organic food consumption or purchase has been documented in several 


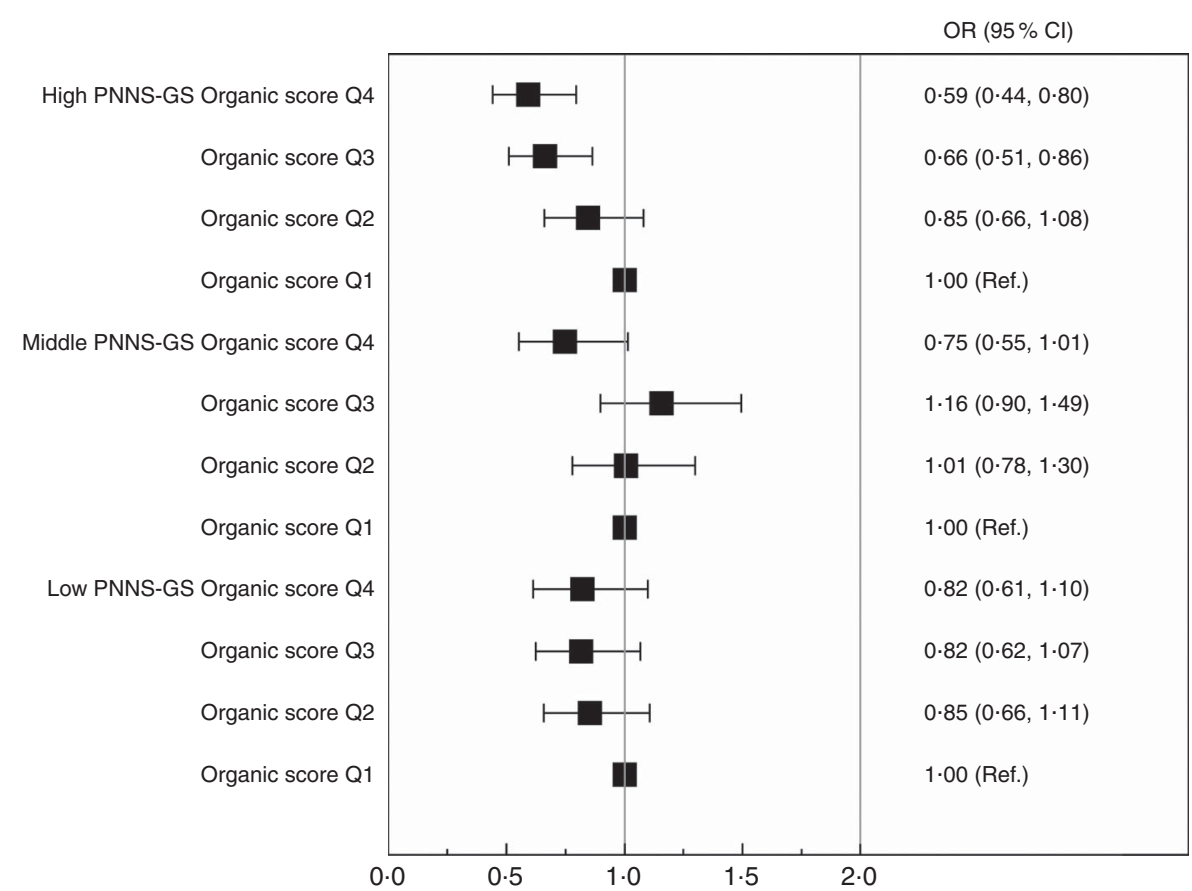

Fig. 2. Prospective association between the organic score in quartiles $(Q)$ and the risk of obesity stratified according to the nutritional quality of the diet, NutriNetSanté, 2009-2015. Values are OR and 95\% Cl using the first quartile (Q1) as the reference, adjusted for age, sex, month and year of inclusion, delay in follow-up, occupation, marital status, education, monthly income per unit, dietary supplement use, modified Programme National Nutrition Santé-guidelines score (mPNNS-GS), principal component analysis-extracted dietary patterns scores, energy intake, physical activity, tobacco status and history of chronic diseases. Ref., referent values.

cross-sectional studies ${ }^{(23,24,38)}$. For instance, a previous investigation of the NutriNet-Santé data ${ }^{(23)}$ has shown that regular organic food consumers showed a markedly lower probability of overweight (excluding obesity) and obesity compared with non-consumers ( -36 and $-62 \%$ in men and -42 and $-48 \%$ in women, respectively). In addition, in the NVS $\mathrm{II}^{(24)}$, as compared with non-buyers of organic food, buyers of organic food had lower proportions of overweight $(35.5 v .39 \cdot 2 \%)$ and obesity $(17 \cdot 9 v \cdot 22 \cdot 5 \%)$. Our findings are also concordant with a small Italian clinical study (including 100 healthy males and fifty males suffering from chronic kidney disease, CKD) that reported a statistically significant reduction in weight among CKD patients after introducing an organic diet for a 2-week period $(85.17$ (SD 13.97) kg at baseline $v .79 \cdot 52$ (SD 10.41) kg after the 2 -week intervention, $P<0 \cdot 05)^{(39)}$.

Overall, the currently available cross-sectional or longitudinal surveys have consistently reported an association between higher organic food consumption and lower BMI. The association with a lower increase in BMI over time that we observed in the present study is of particular interest as it supports a possible role of the organic-based diet in weight management among all subjects, beyond the risk of overweight or obesity among initially normal weight (or underweight) individuals.

\section{Modulating effect of the nutritional quality of the diet}

There is growing evidence supporting the observation that consumers of organic food have a nutritionally healthier dietary pattern as well as other beneficial lifestyles such as lower alcohol consumption, no smoking and a higher physical activity level $^{(23,24,40,41)}$. Thus, we hypothesised that the link between consumption of organic foods and body weight might be modulated by the overall nutritional quality of the diet. Indeed, we found that the strongest reduction in obesity risk (related to a higher consumption frequency of organic foods) was observed among participants with a healthier diet. Conversely, among participants with less healthy diets (susceptible to promote weight gain), the association between consumption frequency of organic food and obesity risk was of smaller magnitude. It is well known that unhealthy eating habits play a key role in the aetiology of obesity ${ }^{(3)}$. Therefore, the mode of production of the foods consumed may be of secondary importance in these subjects. It is also possible that their dietary patterns, which are low in fruits and vegetables, led to less-contaminated diets.

Several hypotheses can be proposed to explain the differences in the risk of obesity between organic food consumers and non-consumers who present a healthy diet. As we adjusted and then stratified for the global nutritional quality of the diet (using the mPNNS-GS score) in our models, it is not possible that our results were biased by the fact that organic food consumers present healthier diets. Other possible explanations include differences in organic food and conventional food with respect to various nutritional compounds.

First, the results of a number of studies argue for a higher concentration of PUFA (especially $n-3$ fatty acids) in organic dairy and meat products and antioxidants (especially vitamin C and phenolic compounds) in organic plant foods ${ }^{(11-13)}$. Although observed differences in nutrient content can vary by about $10-68 \%$ at the food level (not accounted for in our study), it is possible that the overall variations in nutrient intake in an organic diet are sufficient to affect weight management ${ }^{(42,43)}$. However, this remains to be further evaluated. 
Another hypothesis is related to the fact that individuals with higher adherence to the French nutritional guidelines tend to consume more plant-based foods. It is well known that plant foods are frequently contaminated by various pesticide residues (about $45 \%$ of the tested samples in Europe) ${ }^{(44)}$ because they are heavily sprayed with pesticides during conventional agricultural production and storage. This hypothesis is in line with findings of human surveys that have related obesity and type 2 diabetes to pesticide exposure ${ }^{(7,9,10,45,46)}$. Thus, unlike consumption of pesticide-free or only slightly contaminated plant products ${ }^{(11,14,15)}$, high consumption of conventionally grown plant foods may be related to adverse health effects related to higher pesticide exposure.

Indeed, replacing conventional food by organic food has been repeatedly shown to drastically reduce the level of organophosphate residues in human urine ${ }^{(16-20)}$. A specific example of a potential adverse health effect of contaminated fruits and vegetables is that high consumers of conventional or contaminated fruits and vegetables presented low semen quality ${ }^{(47,48)}$. This reinforces the concept that different dietary profiles (with various degrees of intake of contaminated food) lead to different levels of exposure to 'obesogen' chemicals ${ }^{(9)}$, but this hypothesis needs to be investigated in future biomonitoring-based studies that compare organic and conventional diets with various dietary profiles.

\section{Potential mechanistic pathways explaining the association between organic food consumption and body weight}

Our findings may be interpreted in light of reduced exposure to pesticides among organic food consumers. Pesticides (prohibited in organic farming when they are synthetic) often present endocrine-disrupting properties that cause developmental and reproductive abnormalities via the modification of signalling processes ${ }^{(46)}$. Besides, new scientific studies argue that pesticides have a role in metabolic disruption ${ }^{(22)}$, leading to obesity and type 2 diabetes ${ }^{(9)}$. Mechanistic pathways depend on the type of pesticides. Previous studies have shown alterations in glucose and lipid metabolism by organochlorines ${ }^{(49)}$. Moreover, organochlorines have been shown to affect the control of adipogenesis by causing alterations in glucose transport and glycolysis, mitochondrial activity and fatty acid oxidation $^{(49)}$. Organophosphates have been shown to alter carbohydrate and lipid metabolism by disrupting glucose homoeostasis $^{(49)}$. In addition, some pesticides have been shown to affect the regulation of eating behaviour and differentiation of adipocytes ${ }^{(50)}$.

Further studies are necessary to better evaluate metabolic disruption and the 'obesogen' capacity of endocrine-disrupting chemicals contained in conventional foods.

\section{Strengths and limitations}

The main limitation of our study is that it is based on selfreported weight and height data. However, these data have been shown to have good concordance with data from clinical assessments in a validation study ${ }^{(26)}$. This validation study showed high intraclass correlation coefficients, ranging from 0.94 for height to 0.99 for weight, and the concordance for BMI classification was $93 \%$ (sensibility $=88 \%$ and specificity $=$ $99 \%)$. Second, the generalisability of our findings is limited as participants were volunteers involved in a long-term cohort focused on nutrition and health. The individuals included in our study are thus likely to be particularly health conscious. A final limitation pertains to the difficulty to disentangle the role of overall dietary patterns from the role of organic food consumption, despite the extensive adjustment and stratification made. As the design of our study is observational, residual confounding cannot be ruled out. In particular, it is likely that unmeasured or only indirectly measured factors, including genetic factors, ethnicity, environmental factors (e.g. food or built environment) or psychological factors (e.g. occupational stress), may modify the association between organic food consumption and obesity.

Our study also presents important strengths. First, the rich and accurately collected data permitted us to account for a broad range of potential confounders including lifestyles and health outcomes. Moreover, the very large sample size of our study enabled us to conduct statistically powerful stratified analyses. Another important strength is the prospective design of our analysis that implies a high level of evidence. Finally, the availability of accurate dietary data allowed us to adjust for the nutritional quality of the diet, using a validated dietary index.

\section{Conclusion}

This study, based on data collected in a very large prospective cohort, is the first to support a prospective relationship between consumption frequency of organic foods and body weight change, as well as a strong negative association with the risk of overweight and obesity. The overall nutritional quality of the diet may exert a modulating effect in these relationships, with a stronger effect observed among those having a healthy, plantbased diet. Further studies, especially studies based on quantitative organic consumption data taking into account a diversity of dietary profiles (plant based, Western, etc.), are needed to confirm these results. If confirmed, these findings are of major interest from a public health point of view, as they reinforce the need to fine-tune nutritional guidelines by accounting for the mode of food production.

\section{Acknowledgements}

The authors thank Nathalie Arnault, Stephen Besseau, Laurent Bourhis, Julien Allègre, Than Duong Van, Younes Esseddik, Cédric Agaesse, Claudia Chahine, Paul Flanzy, Mac Rakotondrazafy and Fabien Szabo for their technical contribution to the NutriNet-Santé study.

The NutriNet-Santé study is supported by the French Ministry of Health, the French Institute for Health Surveillance, the National Institute for Prevention and Health Education, the Foundation for Medical Research, the National Institute for Health and Medical Research, the National Institute for Agricultural Research, the National Conservatory of Arts and Crafts and the University of Paris 13. 
E. K.-G. conducted the literature review, drafted the manuscript and performed the analyses; E. K.-G., J. B., K. E. A., P. G., S. H. and D. L. were involved in the interpretation of results and critically reviewed the manuscript; and S. H., P. G. and E. K.-G. were responsible for the development of the design and protocol of the study. All the authors read and approved the final manuscript.

None of the authors has any conflicts of interest to declare.

\section{Supplementary material}

For supplementary material/s referred to in this article, please visit https://doi.org/10.1017/S0007114517000058

\section{References}

1. World Health Organization (2000) Obesity: preventing and managing the global epidemic. Report of a WHO consultation. World Health Organ Tech Rep Ser 894, 1-252.

2. World Health Organization (2009) Global health risks. Geneva: WHO Technical Report.

3. World Health Organization Europe (2007) The Challenge of Obesity in the WHO European Region. Copenhagen: WHO Europe.

4. Latino-Martel P, Cottet V, Druesne-Pecollo N, et al. (2016) Alcoholic beverages, obesity, physical activity and other nutritional factors, and cancer risk: a review of the evidence. Crit Rev Oncol Hematol 99, 308-323.

5. American Institute for Cancer Research \& World Cancer Research Fund (2007) Food, Nutrition, Physical Activity, and the Prevention of Cancer: A Global Perspective. Washington, DC: American Institute for Cancer Research.

6. Hansen TH, Gobel RJ, Hansen T, et al. (2015) The gut microbiome in cardio-metabolic health. Genome Med 7, 33.

7. Lee DH, Porta M, Jacobs DR Jr, et al. (2014) Chlorinated persistent organic pollutants, obesity, and type 2 diabetes. Endocr Rev 35, 557-601.

8. Snedeker SM \& Hay AG (2012) Do interactions between gut ecology and environmental chemicals contribute to obesity and diabetes? Environ Health Perspect 120, 332-339.

9. Thayer KA, Heindel JJ, Bucher JR, et al. (2012) Role of environmental chemicals in diabetes and obesity: a National Toxicology Program workshop review. Environ Health Perspect 120, 779-789.

10. Lubrano C, Genovesi G, Specchia P, et al. (2013) Obesity and metabolic comorbidities: environmental diseases? Oxid Med Cell Longev 2013, 640673.

11. Baranski M, Srednicka-Tober D, Volakakis N, et al. (2014) Higher antioxidant and lower cadmium concentrations and lower incidence of pesticide residues in organically grown crops: a systematic literature review and meta-analyses. Br J Nutr 112, 794-811.

12. Srednicka-Tober D, Baranski M, Seal C, et al. (2016) Composition differences between organic and conventional meat: a systematic literature review and meta-analysis. $\mathrm{Br} J \mathrm{Nutr}$ 115, 994-1011.

13. Srednicka-Tober D, Baranski M, Seal CJ, et al. (2016) Higher PUFA and $n-3$ PUFA, conjugated linoleic acid, alphatocopherol and iron, but lower iodine and selenium concentrations in organic milk: a systematic literature review and meta- and redundancy analyses. Br J Nutr 115, 1043-1060.

14. Smith-Spangler C, Brandeau ML, Hunter GE, et al. (2012) Are organic foods safer or healthier than conventional alternatives?: a systematic review. Ann Intern Med 157, 348-366.

15. Lairon D (2010) Nutritional quality and safety of organic food: a review. Agron Sustain Dev 30, 33-41.

16. Curl CL, Fenske RA \& Elgethun K (2003) Organophosphorus pesticide exposure of urban and suburban preschool children with organic and conventional diets. Environ Health Perspect 111, 377-382.

17. Lu C, Toepel K, Irish R, et al. (2006) Organic diets significantly lower children's dietary exposure to organophosphorus pesticides. Environ Health Perspect 114, 260-263.

18. Bradman A, Quiros-Alcala L, Castorina R, et al. (2015) Effect of organic diet intervention on pesticide exposures in young children living in low-income urban and agricultural communities. Environ Health Perspect 123, 1086-1093.

19. Oates L, Cohen M, Braun L, et al. (2014) Reduction in urinary organophosphate pesticide metabolites in adults after a weeklong organic diet. Environ Res 132, 105-111.

20. Curl CL, Beresford SA, Fenske RA, et al. (2015) Estimating pesticide exposure from dietary intake and organic food choices: the Multi-Ethnic Study of Atherosclerosis (MESA). Environ Health Perspect 123, 475-483.

21. Mnif W, Hassine AI, Bouaziz A, et al. (2011) Effect of endocrine disruptor pesticides: a review. Int J Environ Res Public Health 8, 2265-2303.

22. Casals-Casas C \& Desvergne B (2011) Endocrine disruptors: from endocrine to metabolic disruption. Annu Rev Physiol 73, $135-162$.

23. Kesse-Guyot E, Peneau S, Mejean C, et al. (2013) Profiles of organic food consumers in a large sample of French adults: results from the Nutrinet-Sante cohort study. PLOS ONE $\mathbf{8}$, e76998.

24. Eisinger-Watzl M, Wittig F, Heuer T, et al. (2015) Customers purchasing organic food - do they live healthier? Results of the German National Nutrition Survey II. Eur J Nutr Food Saf 5 , 59-71.

25. Hercberg S, Castetbon K, Czernichow S, et al. (2010) The Nutrinet-Sante Study: a web-based prospective study on the relationship between nutrition and health and determinants of dietary patterns and nutritional status. BMC Public Health 10, 242.

26. Lassale C, Peneau S, Touvier M, et al. (2013) Validity of webbased self-reported weight and height: results of the NutrinetSante study. J Med Internet Res 15, e152.

27. Lassale C, Castetbon K, Laporte F, et al. (2015) Validation of a Web-based, self-administered, non-consecutive-day dietary record tool against urinary biomarkers. Br J Nutr 113, 953-962.

28. Lassale C, Castetbon K, Laporte F, et al. (2015) Correlations between fruit, vegetables, fish, vitamins, and fatty acids estimated by web-based nonconsecutive dietary records and respective biomarkers of nutritional status. J Acad Nutr Diet 116, 427-438.e5.

29. Le Moullec N, Deheeger M, Preziosi P, et al. (1996) Validation du manuel photos utilisé pour l'enquête alimentaire de l'étude SU.VI.MAX (Validation of the photo manual used for the collection of dietary data in the SU.VI.MAX study). Cah Nutr Diét 31, 158-164.

30. NutriNet-Santé Coordination (2013) Table de composition des aliments - Etude NutriNet-Santé. Paris: Economica.

31. Black AE (2000) Critical evaluation of energy intake using the Goldberg cut-off for energy intake:basal metabolic rate. A practical guide to its calculation, use and limitations. Int J Obes Relat Metab Disord 24, 1119-1130.

32. Estaquio C, Kesse-Guyot E, Deschamps V, et al. (2009) Adherence to the French Programme National Nutrition Sante 
Guideline Score is associated with better nutrient intake and nutritional status. J Am Diet Assoc 109, 1031-1041.

33. Institut National de la Statistique et des Etudes Economiques (2012) Definition of the consumption unit. https://www.insee. $\mathrm{fr} / \mathrm{fr} /$ metadonnees/definition/c1802 (accessed January 2016).

34. Hagstromer M, Oja P \& Sjostrom M (2006) The International Physical Activity Questionnaire (IPAQ): a study of concurrent and construct validity. Public Health Nutr 9, 755-762.

35. Ali MS, Groenwold RH \& Klungel OH (2016) Best (but oftforgotten) practices: propensity score methods in clinical nutrition research. Am J Clin Nutr 104, 247-258.

36. Seaman SR \& White IR (2011) Review of inverse probability weighting for dealing with missing data. Stat Methods Med Res 22, 278-295.

37. Baudry J, Mejean C, Peneau S, et al. (2015) Health and dietary traits of organic food consumers: results from the NutriNetSante study. Br J Nutr 114, 2064-2073.

38. Torjusen H, Brantsaeter AL, Haugen M, et al. (2010) Characteristics associated with organic food consumption during pregnancy; data from a large cohort of pregnant women in Norway. BMC Public Health 10, 775.

39. De Lorenzo A, Noce A, Bigioni M, et al. (2010) The effects of Italian Mediterranean organic diet (IMOD) on health status. Curr Pharm Des 16, 814-824.

40. Oates L, Cohen M \& Braun L (2012) Characteristics and consumption patterns of Australian organic consumers. $J$ Sci Food Agric 92, 2782-2787.

41. Petersen SB, Rasmussen MA, Strom M, et al. (2013) Sociodemographic characteristics and food habits of organic consumers - a study from the Danish National Birth Cohort. Public Health Nutr 16, 1810-1819.
42. Lorente-Cebrian S, Costa AG, Navas-Carretero S, et al. (2013) Role of omega-3 fatty acids in obesity, metabolic syndrome, and cardiovascular diseases: a review of the evidence. J Physiol Biochem 69, 633-651.

43. Meydani M \& Hasan ST (2010) Dietary polyphenols and obesity. Nutrients $\mathbf{2}, 737-751$.

44. European Food Safety Authority (2015) The 2013 European Union Report on Pesticide Residues in Food. EFSAJ 13, 4038 .

45. Lee DH, Steffes MW, Sjodin A, et al. (2011) Low dose organochlorine pesticides and polychlorinated biphenyls predict obesity, dyslipidemia, and insulin resistance among people free of diabetes. PLOS ONE 6, e15977.

46. Heindel JJ, Newbold R \& Schug TT (2015) Endocrine disruptors and obesity. Nat Rev Endocrinol 11, 653-661.

47. Chiu YH, Afeiche MC, Gaskins AJ, et al. (2015) Fruit and vegetable intake and their pesticide residues in relation to semen quality among men from a fertility clinic. Hum Reprod 30, 1342-1351.

48. Juhler RK, Larsen SB, Meyer O, et al. (1999) Human semen quality in relation to dietary pesticide exposure and organic diet. Arch Environ Contam Toxicol 37, 415-423.

49. Androutsopoulos VP, Hernandez AF, Liesivuori J, et al. (2013) A mechanistic overview of health associated effects of low levels of organochlorine and organophosphorous pesticides. Toxicology 307, 89-94.

50. Mostafalou S \& Abdollahi M (2013) Pesticides and human chronic diseases: evidences, mechanisms, and perspectives. Toxicol Appl Pharmacol 268, 157-177. 